\title{
IMPULSIVE DIFFUSION EQUATION ON TIME SCALES
}

\section{TUBA GULSEN, SHAIDA SABER MAWLOOD SIAN, EMRAH YILMAZ* AND HIKMET KOYUNBAKAN}

\author{
Department of Mathematics, Faculty of Science, Firat University, 23119, Elazig, Turkey
}

*Corresponding author: emrah231983@gmail.com

\begin{abstract}
Application of boundary value problems (BVP's) on an arbitrary time scale $\mathbb{T}$ is a fairly new and important subject in mathematics. In this study, we deal with an eigenvalue problem for impulsive diffusion equation with boundary conditions on $\mathbb{T}$. We generalize some noteworthy results about spectral theory of classical diffusion equation into $\mathbb{T}$. Also, some eigenfunction estimates of the impulsive diffusion eigenvalue problem are established on $\mathbb{T}$.
\end{abstract}

\section{INTRODUCTION}

Time scale theory was first considered by Stefan Hilger [1] in 1988 in his doctoral dissertation under supervision of Bernard Aulbach [2] to unify the two approaches of dynamic modelling: difference and differential equations. However, similar ideas have been used before and go back at least to the introduction of the Rieamann-Stieltjes integral which unifies sums and integrals. More specifically, $\mathbb{T}$ is an arbitrary, non-empty, closed subset of $\mathbb{R}$. Many results related to differential equations carry over quite easily to corresponding results for difference equations, while other results seem to be totally different in nature. Because of these reasons, the theory of dynamic equations is an active area of research. The time scale calculus can be applied to any fields in which dynamic processes are described by discrete or continuous time models. So, the calculus of time scale has various applications involving non-continuous domains like modeling of certain bug populations, chemical reactions, phytoremediation of metals, wound healing, maximization problems in

Received $3^{\text {rd }}$ October, 2017; accepted $8^{\text {th }}$ December, 2017; published $3^{\text {rd }}$ January, 2018.

2010 Mathematics Subject Classification. 34N05, 34L40, 34L05.

Key words and phrases. time scales; impulsive diffusion equation; spectral theory.

(C)2018 Authors retain the copyrights of their papers, and all open access articles are distributed under the terms of the Creative Commons Attribution License. 
economics and traffic problems. In recent years, several authors have obtained many important results about different topics on time scales (see [3-7]).

Although there are many studies in the literature on time scales, very little work has been done about BVP's. The first studies on these type problems for linear $\Delta$ - differential equations on $\mathbb{T}$ were fulfilled by Chyan, Davis, Henderson and Yin [8] in 1998 and Agarwal, Bohner and Wong [9] in 1999. In [8], the theory of positive operators according to a cone in a Banach space is applied to eigenvalue problems related to the second order linear $\Delta$-differential equations on $\mathbb{T}$ to prove existence of a smallest positive eigenvalue and then a theorem proved to compare the smallest positive eigenvalue for two problems of that type. In [9], an oscillation theorem is given for Sturm-Liouville (SL) eigenvalue problem on $\mathbb{T}$ with separated boundary conditions and Rayleigh's principle is studied. In 2002, Agarwal, Bohner and O'Regan [10] presented some new existence results for time scale BVP's on infinite intervals. Guseinov [11] investigated some eigenfunction expansions for the simple SL eigenvalue problem on $\mathbb{T}$ in 2007 . In that paper, the existence of the eigenvalues and eigenfunctions is proved and mean square convergent and uniformly convergent expansions in eigenfunctions are established by Guseinov. Later, Huseynov and Bairamov generalized the results of Guseinov to the more general eigenvalue problem in 2009 [12]. Zhang and Ma investigated solvability of SL problems on $\mathbb{T}$ at resonance in 2010 [13]. Guo Zhang and Sun [14] gave a variational approach for SL BVP's on $\mathbb{T}$ in 2011. Erbe, Mert and Peterson [15] derived formulas for finding two linearly independent solutions of the SL dynamic equation in 2012. Yilmaz, Koyunbakan and Ic gave some substantial results for diffusion equation on $\mathbb{T}$ in 2015 [16]. Yaslan [17] proved the existence of positive solutions for second-order impulsive BVP's on $\mathbb{T}$ in 2016. Allahverdiev, Eryilmaz and Tuna [18] considered dissipative SL operators with a spectral parameter under the boundary condition on bounded time scales in 2017. Gulsen and Yilmaz [19] explained some properties of Dirac system on time scales in 2017.

As can be seen from the literature, the studies about spectral theory on time scales have focused on SL equation and Dirac system. We notice that there isn't any research about impulsive diffusion equation on time scales according to our survey. Hence, this study will be the first related to spectral theory of impulsive diffusion equation on $\mathbb{T}$. Our study will give a lead to mathematicians to solve some direct and inverse spectral problems for any types of operators on $\mathbb{T}$. To give basic results, we need to remind some fundamental concepts on time scales.

Forward and backward jump operators at $t \in \mathbb{T}$, for $t<\sup \mathbb{T}$ are defined as

$$
\sigma(t)=\inf \{s \in \mathbb{T}: s>t\}, \rho(t)=\sup \{s \in \mathbb{T}: s<t\}
$$

respectively ( $\operatorname{supplemented~by~} \inf \phi=\sup \mathbb{T}$ and $\sup \phi=\inf \mathbb{T}$ where $\phi$ indicates the empty set). These operators are well-defined and map $\mathbb{T}$ into $\mathbb{T}$. At the same time, $t$ is said to be left dense, left scattered, right dense and right scattered if $\rho(t)=t, \rho(t)<t, \sigma(t)=t$ and $\sigma(t)>t$, respectively. The distance from an 
arbitrary element $t \in \mathbb{T}$ to the closest element on the right is called graininess of $\mathbb{T}$ and is determined by

$$
\mu(t)=\sigma(t)-t
$$

A closed interval on $\mathbb{T}$ is defined by

$$
[a, b]_{\mathbb{T}}=\{t \in \mathbb{T}: a \leq t \leq b\}
$$

where $a$ and $b$ are fixed points of $\mathbb{T}$ with $a<b$. For standard notions and notations related to time scales theory, we refer to the reference [3].

We also need to explain $\mathbb{T}^{K}$ along with the set $\mathbb{T}$ to define $\Delta$ - derivative of a function. If $\mathbb{T}$ has left scattered maximum $m$, then $\mathbb{T}^{K}=\mathbb{T}-\{m\}$. Otherwise, we put $\mathbb{T}^{K}=\mathbb{T}[3]$. Assume $y: \mathbb{T} \rightarrow \mathbb{R}$ is a function and $t \in \mathbb{T}^{K}$. Then, we define $y^{\Delta}(t)$ to be the number (provided it exists) with the property that given any $\varepsilon>0$, there is a neighborhood $U=(t-\delta, t+\delta) \cap \mathbb{T}$ of $t$ for some $\delta>0$ such that

$$
\left|[y(\sigma(t))-y(s)]-y^{\Delta}(t)[\sigma(t)-s]\right| \leq \varepsilon|\sigma(t)-s|
$$

for all $s \in U$. We call $y^{\Delta}(t)$ as the delta $(\Delta)$ or Hilger derivative of $y$ at $t$. The function $y: \mathbb{T} \rightarrow \mathbb{R}$ is called $r d$-continuous if it is continuous at right dense points on $\mathbb{T}$ and its left sided limits exist (finite) at left dense points on $\mathbb{T}$. The set of all $r d$-continuous functions on $\mathbb{T}$ is denoted by $C_{r d}(\mathbb{T})$. The set of all functions that are $\Delta$-differentiable and whose $\Delta$-derivative is $r d$-continuous on $\mathbb{T}$ is denoted by $C_{r d}^{1}(\mathbb{T})$. However, $y$ is regulated if its right sided limits exist (finite) at all right dense points on $\mathbb{T}$ and its left sided limits exist (finite) at all left dense points on $\mathbb{T}$. Let $y$ be a regulated function on $\mathbb{T}$. The indefinite $\Delta$-integral of $y$ is denoted by

$$
\int y(t) \Delta t=Y(t)+C,
$$

where $C$ is an arbitrary constant and $Y$ is pre-antiderivative of $y$. Finally, definite $\Delta-$ integral of $y$ is defined by

$$
\int_{r}^{s} y(t) \Delta t=Y(s)-Y(r)
$$

for all $r, s \in \mathbb{T}$.

Here, we want to state some major spectral properties of impulsive diffusion eigenvalue problem of the form

$$
L y=-y^{\Delta \Delta}(t)+\{q(t)+2 \lambda p(t)\} y^{\sigma}(t)=\lambda^{2} \varpi(t) y^{\sigma}(t), t \in[\rho(a), b] \cap \mathbb{T}
$$

with the boundary conditions

$$
\begin{aligned}
y^{\Delta}(\rho(a)) & =0, \\
\alpha y(b)+\beta y^{\Delta}(b) & =0,
\end{aligned}
$$


where $\lambda$ is a spectral parameter and

$$
\varpi(t)=\left\{\begin{array}{cc}
1, & \rho(a)<t<\frac{b}{2} \\
\vartheta^{2}, & \frac{b}{2}<t<b
\end{array} \quad, 0<\vartheta \neq 1 .\right.
$$

We suppose that $p, q:[\rho(a), b] \cap \mathbb{T} \rightarrow \mathbb{R}$ are continuous functions; $a, b \in \mathbb{T}$ with $a<b, y^{\sigma}=y(\sigma)$ and $\left(\alpha^{2}+\beta^{2}\right) \neq 0 . \quad y(t)$ is known as eigenfunction of the problem (1.1)-(1.3). By taking $\mathbb{T}=\mathbb{R}$ in $(1.1)$, we obtain below classical impulsive diffusion equation (or quadratic pencil of Schrödinger equation)

$$
-y^{\prime \prime}(t)+\{q(t)+2 \lambda p(t)\} y(t)=\lambda^{2} \varpi(t) y(t)
$$

(1.4) is reduced to Sturm-Liouville equation when $p(t)=0$ and $\varpi(t)=1$ [20]. Now, we give some information related to the physical meaning and historical development of diffusion equation in classical spectral theory.

The problem of defining the interactions among colliding particles is an attractive issue in mathematical physics. In many cases, a definition can be carried out through a well known theoretical model. Particularly, one is interested in collisions of two spinless particles, and it is assumed that the $s$-wave binding energies and $s$-wave scattering matrix are completely known from collision experiments. $s$-wave Schrödinger equation with a radial static potential $V$ can be defined by

$$
y^{\prime \prime}+[E-V(x)] y=0, x \geq 0
$$

where $V$ depends on energy and has the following energy dependence

$$
V(x, E)=U(x)+2 \sqrt{E} Q(x) .
$$

$U(x)$ and $Q(x)$ are complex-valued functions. (1.5) reduces to the Klein-Gordon $s$-wave equation with the static potential $Q(x)$, for a particle of zero mass and the energy $\sqrt{E}$ with the supplementary condition $U(x)=-Q^{2}(x)$ [21]. The Klein-Gordon equation is an attempt to marry special relativity and quantum mechanics. It is derived from Einstein's energy equation where the energy and momentum terms are replaced with quantum mechanical operators. The Klein-Gordon equation describes particles of spin-0 hence it describes the Higgs boson. However, it is not positive definite because it is a second order differential equation and when considering its interpretation from within a quantum mechanical framework we run into trouble namely negative probability densities, which are not allowed in quantum mechanics [22].

Jaulent and Jean [21] explained the actual background of diffusion operator in 1972. Maksudov and Guseinov [23] considered the spectral theory of diffusion operator in 1979. Then, Gasymov and Guseinov [24] determined the diffusion operator from spectral data in 1981. Fragela [25] studied on this operator with integral boundary conditions in 1984. Maksudov and Guseinov [26] gave some solutions of the inverse scattering problem for diffusion operator on whole axis in 1986. Bairamov, Çakar and Celebi [27] obtained some results for diffusion operator with spectral singularities, disctrete spectrum and principal functions 
in 1997. Moreover, inverse nodal problem was first considered by Koyunbakan [28] in 2006 for diffusion operator. Koyunbakan [29] obtained uniqueness results and reconstruction formulas in 2007 with Panakhov. Koyunbakan and Yilmaz [30] expressed reconstruction formulas for the $k$-th derivatives of the first potential function $q$ in diffusion equation by using nodal points in 2008. Yang [31] extended their results to the second potential function $p$ in 2010. Yang and Zettl [32] solved half-inverse diffusion problem in 2012. We can replicate these examples. There are many other researches on diffusion operator in literature by many authors (see [33-42]).

This study is collocated as follows: In Section 2, we prove some basic theorems for impulsive diffusion equation on $\mathbb{T}$. Using some methods, we get asymptotic estimate of eigenfunction for the problem (1.1)-(1.3) in Section 3. In Section 4, we give a conclusion to summarize our study.

\section{Some spectral properties of Impulsive Diffusion Equation on time scales}

It is well known that the problem (1.1)-(1.3) has only real, simple eigenvalues and its eigenfunctions are orthogonal when $\mathbb{T}=\mathbb{R}[24]$. The below results will generalize this basic consequences to an arbitrary time scale.

Theorem 2.1. The eigenvalues of the problem (1.1)-(1.3) are all simple.

Proof. Let $\lambda \in \mathbb{R}$ be spectral parameter and $y_{1}(t), y_{2}(t)$ be eigenfunctions of (1.1)-(1.3). Then, we have

$$
\begin{aligned}
& L y_{1}=-y_{1}^{\Delta \Delta}(t)+\{q(t)+2 \lambda p(t)\} y_{1}^{\sigma}(t)=\lambda^{2} \varpi(t) y_{1}^{\sigma}(t), \\
& L y_{2}=-y_{2}^{\Delta \Delta}(t)+\{q(t)+2 \lambda p(t)\} y_{2}^{\sigma}(t)=\lambda^{2} \varpi(t) y_{2}^{\sigma}(t) .
\end{aligned}
$$

Multiplying these equations by $y_{2}^{\sigma}$ and $y_{1}^{\sigma}$ respectively and substracting, we get

$$
\left(y_{1} y_{2}^{\Delta}-y_{2} y_{1}^{\Delta}\right)^{\Delta}=0
$$

By taking $\Delta$-integral of $(2.1)$ on $[\rho(a), b]$, we conclude

$$
\left.W\left[y_{1}, y_{2}\right]\right|_{\rho(a)} ^{b}=c
$$

Using the conditions (1.2), (1.3), one can easily obtain that $c=0$. Then

$$
\left(\frac{y_{1}}{y_{2}}\right)^{\Delta}=0
$$

The last equality means that $y_{1}=k y_{2}(k$ constant $)$ and it implies $y_{1}$ and $y_{2}$ are linearly dependent eigenfunctions of (1.1)-(1.3). This completes the proof.

Theorem 2.2. All eigenvalues of the problem (1.1)-(1.3) are all real. 
Proof. Let $\bar{\lambda}_{0}$ be a complex eigenvalue and $\bar{y}(t)$ be an eigenfunction of the problem (1.1)-(1.3) related to $\bar{\lambda}_{0}$. Then, we obtain

$$
\begin{aligned}
\left\{y^{\Delta} \bar{y}-\bar{y}^{\Delta} y\right\}^{\Delta} & =y^{\Delta \Delta} \bar{y}^{\sigma}-\bar{y}^{\Delta \Delta} y^{\sigma} \\
& =\left\{q(t)+2 \lambda_{0} p(t)-\lambda_{0}^{2} \varpi(t)\right\} y^{\sigma} \bar{y}^{\sigma}-\left\{q(t)+2 \bar{\lambda}_{0} p(t)-\bar{\lambda}_{0}^{2} \varpi(t)\right\} \bar{y}^{\sigma} y^{\sigma} \\
& =\left(\bar{\lambda}_{0}^{2}-\lambda_{0}^{2}\right) \varpi(t) y^{\sigma} \bar{y}^{\sigma}-2\left(\bar{\lambda}_{0}-\lambda_{0}\right) p(t) y^{\sigma} \bar{y}^{\sigma} \\
& =\left(\bar{\lambda}_{0}^{2}-\lambda_{0}^{2}\right) \varpi(t)\left|y^{\sigma}(t)\right|^{2}-2\left(\bar{\lambda}_{0}-\lambda_{0}\right) p(t)\left|y^{\sigma}(t)\right|^{2}
\end{aligned}
$$

If we take $\Delta$-integral of the last equality from $\rho(a)$ to $b$, we get

$$
\begin{aligned}
\left(\bar{\lambda}_{0}^{2}-\lambda_{0}^{2}\right) \int_{\rho(a)}^{b} \varpi(t)\left|y^{\sigma}(t)\right|^{2} \Delta t-2\left(\bar{\lambda}_{0}-\lambda_{0}\right) \int_{\rho(a)}^{b} p(t)\left|y^{\sigma}(t)\right|^{2} \Delta t & =y^{\Delta}(b) \bar{y}(b)-\bar{y}^{\Delta}(b) y(b) \\
& -y^{\Delta}(\rho(a)) \bar{y}(\rho(a))+\bar{y}^{\Delta}(\rho(a)) y(\rho(a)) \\
& =0,
\end{aligned}
$$

by considering the conditions (1.2), (1.3). So, we have

$$
\left(\bar{\lambda}_{0}+\lambda_{0}\right) \int_{\rho(a)}^{b} \varpi(t)\left|y^{\sigma}(t)\right|^{2} \Delta t-2 \int_{\rho(a)}^{b} p(t)\left|y^{\sigma}(t)\right|^{2} \Delta t=0 \Rightarrow y^{\sigma}(t)=0,
$$

where $\left(\bar{\lambda}_{0}+\lambda_{0}\right) \varpi(t)-2 p(t)>0$ for $\bar{\lambda}_{0} \neq \lambda_{0}$. This is a contradiction. Hence, eigenvalues of the problem (1.1)-(1.3) are all real.

Theorem 2.3. Let $y_{1}, y_{2} \in C_{r d}^{1}(\mathbb{T})$ be the eigenfunctions of the problem (1.1)-(1.3). Then,

a) $\left(L y_{1}\right) y_{2}^{\sigma}-\left(L y_{2}\right) y_{1}^{\sigma}=W^{\Delta}\left(y_{1}, y_{2}\right)$ on $[\rho(a), b] \cap \mathbb{T}$.

b) $<L y_{1}, y_{2}^{\sigma}>-<L y_{2}, y_{1}^{\sigma}>=W\left(y_{1}, y_{2}\right)(b)-W\left(y_{1}, y_{2}\right)(\rho(a))$,

where $W\left(y_{1}, y_{2}\right)=y_{1} y_{2}^{\Delta}-y_{2} y_{1}^{\Delta}$ is the Wronskian of the functions $y_{1}$ and $y_{2}$.

Proof. a) Definition of the Wronskian and product rule for $\Delta$-derivative give following result:

$$
\begin{aligned}
W^{\Delta}\left(y_{1}, y_{2}\right) & =y_{2}^{\Delta \Delta} y_{1}^{\sigma}-y_{1}^{\Delta \Delta} y_{2}^{\sigma} \\
& =-y_{1}^{\sigma}\left(-y_{2}^{\Delta \Delta}+(q(t)+2 \lambda p(t)) y_{2}^{\sigma}\right)+y_{2}^{\sigma}\left(-y_{1}^{\Delta \Delta}+(q(t)+2 \lambda p(t)) y_{1}^{\sigma}\right) \\
& =\left(L y_{1}\right) y_{2}^{\sigma}-\left(L y_{2}\right) y_{1}^{\sigma} .
\end{aligned}
$$


b) By using definition of Wronskian and inner product on the set of so-called $r d$-continuous functions, we have

$$
\begin{aligned}
<L y_{1}, y_{2}^{\sigma}>-<L y_{2}, y_{1}^{\sigma}>=\int_{\rho(a)}^{b}\left[\left(L y_{1}\right) y_{2}^{\sigma}-\left(L y_{2}\right) y_{1}^{\sigma}\right] \Delta t \\
=\int_{\rho(a)}^{b}\left\{\left(-y_{1}^{\Delta \Delta}+(q(t)+2 \lambda p(t)) y_{1}^{\sigma}\right) y_{2}^{\sigma}-\left(-y_{2}^{\Delta \Delta}+(q(t)+2 \lambda p(t)) y_{2}^{\sigma}\right) y_{1}^{\sigma}\right\} \Delta t \\
=-\int_{\rho(a)}^{b}\left\{y_{1}^{\Delta \Delta} y_{2}^{\sigma}-y_{2}^{\Delta \Delta} y_{1}^{\sigma}\right\} \Delta t \\
=\int_{\rho(a)}^{b}\left\{W\left(y_{1}, y_{2}\right)\right\}^{\Delta} \Delta t \\
=W\left(y_{1}, y_{2}\right)(b)-W\left(y_{1}, y_{2}\right)(\rho(a)) .
\end{aligned}
$$

Hence, this completes the proof. In classical spectral theory, these equalities are known as Lagrange's identity and Green's formula, respectively.

Theorem 2.4. The equality

$$
y(t, \lambda) \frac{\partial}{\partial \lambda} y^{\Delta}(t, \lambda)-y^{\Delta}(t, \lambda) \frac{\partial}{\partial \lambda} y(t, \lambda)=-2 \lambda \int_{\rho(a)}^{t} \varpi(\tau)\left(y^{\sigma}(\tau, \lambda)\right)^{2} \Delta \tau+2 \int_{\rho(a)}^{t} p(\tau)\left(y^{\sigma}(\tau, \lambda)\right)^{2} \Delta \tau
$$

holds for all $t \in[\rho(a), b] \cap \mathbb{T}$ and $\lambda \in \mathbb{R}$.

Proof. Let $\gamma, \lambda \in \mathbb{R}$ with $\gamma \neq \lambda$. Then,

$$
\begin{aligned}
\left\{y(t, \gamma) y^{\Delta}(t, \lambda)-y^{\Delta}(t, \gamma) y(t, \lambda)\right\}^{\Delta} & =y^{\sigma}(t, \gamma) y^{\Delta \Delta}(t, \lambda)-y^{\Delta \Delta}(t, \gamma) y^{\sigma}(t, \lambda) \\
& =y^{\sigma}(t, \gamma)\left\{q(t)+2 \lambda p(t)-\lambda^{2} \varpi(t)\right\} y^{\sigma}(t, \lambda) \\
& -y^{\sigma}(t, \lambda)\left\{q(t)+2 \gamma p(t)-\gamma^{2} \varpi(t)\right\} y^{\sigma}(t, \gamma) \\
& =\left(\gamma^{2}-\lambda^{2}\right) \varpi(t) y^{\sigma}(t, \lambda) y^{\sigma}(t, \gamma)-2(\gamma-\lambda) p(t) y^{\sigma}(t, \lambda) y^{\sigma}(t, \gamma) .
\end{aligned}
$$

Dividing both sides of above equality by $\lambda-\gamma$ and taking limit as $\gamma \rightarrow \lambda$, we have

$$
\begin{gathered}
\lim _{\gamma \rightarrow \lambda} \frac{\left\{y(t, \gamma) y^{\Delta}(t, \lambda)-y^{\Delta}(t, \gamma) y(t, \lambda)\right\}^{\Delta}}{\lambda-\gamma}=-\lim _{\gamma \rightarrow \lambda}\left\{(\gamma+\lambda) \varpi(t) y^{\sigma}(t, \lambda) y^{\sigma}(t, \gamma)-2 p(t) y^{\sigma}(t, \lambda) y^{\sigma}(t, \gamma)\right\} \\
\Rightarrow\left\{y(t, \lambda) \frac{\partial}{\partial \lambda} y^{\Delta}(t, \lambda)-y^{\Delta}(t, \lambda) \frac{\partial}{\partial \lambda} y(t, \lambda)\right\}^{\Delta}=-2 \lambda \varpi(t)\left(y^{\sigma}(t, \lambda)\right)^{2}+2 p(t)\left(y^{\sigma}(t, \lambda)\right)^{2} .
\end{gathered}
$$


By taking $\Delta$-integral of the last equality from $\rho(a)$ to $t$, we get

$$
\begin{aligned}
\int_{\rho(a)}^{t}\left\{y(\tau, \lambda) \frac{\partial}{\partial \lambda} y^{\Delta}(\tau, \lambda)-y^{\Delta}(\tau, \lambda) \frac{\partial}{\partial \lambda} y(\tau, \lambda)\right\}^{\Delta} \Delta \tau & =-2 \lambda \int_{\rho(a)}^{t} \varpi(\tau)\left(u^{\sigma}(\tau, \lambda)\right)^{2} \Delta \tau \\
& +2 \int_{\rho(a)}^{t} p(\tau)\left(u^{\sigma}(\tau, \lambda)\right)^{2} \Delta \tau .
\end{aligned}
$$

Since $y(\rho(a), \lambda)=1$ and $y^{\Delta}(\rho(a), \lambda)=0$, it yields $\frac{\partial}{\partial \lambda} y(\rho(a), \lambda)=0$ and $\frac{\partial}{\partial \lambda} y^{\Delta}(\rho(a), \lambda)=0$. Finally, after some computations, we obtain

$$
y(t, \lambda) \frac{\partial}{\partial \lambda} y^{\Delta}(t, \lambda)-y^{\Delta}(t, \lambda) \frac{\partial}{\partial \lambda} y(t, \lambda)=-2 \lambda \int_{\rho(a)}^{t} \varpi(\tau)\left(u^{\sigma}(\tau, \lambda)\right)^{2} \Delta \tau+2 \int_{\rho(a)}^{t} p(\tau)\left(u^{\sigma}(\tau, \lambda)\right)^{2} \Delta \tau .
$$

So, the proof is complete.

Theorem 2.5. The eigenfunctions $y_{1}\left(t, \lambda_{1}\right)$ and $y_{2}\left(t, \lambda_{2}\right)$ of the problem (1.1)-(1.3) corresponding to distinct eigenvalues $\lambda_{1}$ and $\lambda_{2}$ are orthogonal, i.e

$$
\left(\lambda_{1}+\lambda_{2}\right) \int_{\rho(a)}^{b} \varpi(t) y_{1}^{\sigma}\left(t, \lambda_{1}\right) y_{2}^{\sigma}\left(t, \lambda_{2}\right) \Delta t-2 \int_{\rho(a)}^{b} p(t) y_{1}^{\sigma}\left(t, \lambda_{1}\right) y_{2}^{\sigma}\left(t, \lambda_{2}\right) \Delta t=0 .
$$

Proof. Let us use following equality

$$
\left\{y_{1}^{\Delta}\left(t, \lambda_{1}\right) y_{2}\left(t, \lambda_{2}\right)-y_{2}^{\Delta}\left(t, \lambda_{2}\right) y_{1}\left(t, \lambda_{1}\right)\right\}^{\Delta}=\left\{\left(\lambda_{2}^{2}-\lambda_{1}^{2}\right) \varpi(t)-2\left(\lambda_{2}-\lambda_{1}\right) p(t)\right\} y_{1}^{\sigma}\left(t, \lambda_{1}\right) y_{2}^{\sigma}\left(t, \lambda_{2}\right) .
$$

Taking $\Delta$-integral of the last equality from $\rho(a)$ to $b$, we get

$$
\begin{aligned}
\int_{\rho(a)}^{b}\left\{y_{1}^{\Delta}\left(t, \lambda_{1}\right) y_{2}\left(t, \lambda_{2}\right)-y_{2}^{\Delta}\left(t, \lambda_{2}\right) y_{1}\left(t, \lambda_{1}\right)\right\}^{\Delta} \Delta t & =\left(\lambda_{2}^{2}-\lambda_{1}^{2}\right) \int_{\rho(a)}^{b} \varpi(t) y_{1}^{\sigma}\left(t, \lambda_{1}\right) y_{2}^{\sigma}\left(t, \lambda_{2}\right) \Delta t \\
& -2\left(\lambda_{2}-\lambda_{1}\right) \int_{\rho(a)}^{b} p(t) y_{1}^{\sigma}\left(t, \lambda_{1}\right) y_{2}^{\sigma}\left(t, \lambda_{2}\right) \Delta t \\
& =y_{1}^{\Delta}\left(b, \lambda_{1}\right) y_{2}\left(b, \lambda_{2}\right)-y_{2}^{\Delta}\left(b, \lambda_{2}\right) y_{1}\left(b, \lambda_{1}\right) \\
& -y_{1}^{\Delta}\left(\rho(a), \lambda_{1}\right) y_{2}\left(\rho(a), \lambda_{2}\right)+y_{2}^{\Delta}\left(\rho(a), \lambda_{2}\right) y_{1}\left(\rho(a), \lambda_{1}\right) \\
& =0,
\end{aligned}
$$

and

$$
\left(\lambda_{1}+\lambda_{2}\right) \int_{\rho(a)}^{b} \varpi(t) y_{1}^{\sigma}\left(t, \lambda_{1}\right) y_{2}^{\sigma}\left(t, \lambda_{2}\right) \Delta t-2 \int_{\rho(a)}^{b} p(t) y_{1}^{\sigma}\left(t, \lambda_{1}\right) y_{2}^{\sigma}\left(t, \lambda_{2}\right) \Delta t=0,
$$

for $\lambda_{1} \neq \lambda_{2}$. Then, it shows that the eigenfunctions $y_{1}\left(t, \lambda_{1}\right)$ and $y_{2}\left(t, \lambda_{2}\right)$ corresponding to distinct eigenvalues are always orthogonal. 


\section{MAin Results}

In this section, we acquire asymptotic estimate for the eigenfunction $y(t, \lambda)$ of the problem (1.1)-(1.3) on $\mathbb{T}$ whose all points are right dense.

Theorem 3.1. The eigenfunction $y(t, \lambda)$ and its $\Delta$-derivative of the problem (1.1)-(1.3) have the following asymptotic estimates;

$$
y(t, \lambda)=\cos _{\lambda}(t, \rho(a))-\frac{1}{\lambda} \int_{\rho(a)}^{t}(q(s)+2 \lambda p(s)) y(s, \lambda) \sin _{\lambda}(t, s) \Delta s,
$$

and

$$
y^{\Delta}(t, \lambda)=-\lambda \sin _{\lambda}(t, \rho(a))-\int_{\rho(a)}^{t}(q(s)+2 \lambda p(s)) y(s, \lambda) \cos _{\lambda}(t, s) \Delta s,
$$

where $t \in[\rho(a), b] \cap \mathbb{T}$.

Proof. By equation (1.1), we can write

$$
\begin{aligned}
\frac{1}{\lambda} \int_{\rho(a)}^{t}(q(s)+2 \lambda p(s)) y(s, \lambda) \sin _{\lambda}(t, s) \Delta s & =\frac{1}{\lambda} \int_{\rho(a)}^{t} \sin _{\lambda}(t, s)\left(y^{\Delta \Delta}(s, \lambda)+\lambda^{2} \varpi(s) y(s, \lambda)\right) \Delta s \\
& =\frac{1}{\lambda} \int_{\rho(a)}^{t} \sin _{\lambda}(t, s) y^{\Delta \Delta}(s, \lambda) \Delta s+\lambda \int_{\rho(a)}^{t} \sin _{\lambda}(t, s) \varpi(s) y(s, \lambda) \Delta s .
\end{aligned}
$$

After using integration by parts twice for the first integral in (3.1), we get

$$
\begin{aligned}
\frac{1}{\lambda} \int_{\rho(a)}^{t} \sin _{\lambda}(t, s) y^{\Delta \Delta}(s, \lambda) \Delta s & =\left.\frac{1}{\lambda}\left(\sin _{\lambda}(t, s) y^{\Delta}(s, \lambda)\right)\right|_{\rho(a)} ^{t}-\frac{1}{\lambda} \int_{\rho(a)}^{t} \lambda \cos _{\lambda}(t, s) y^{\Delta}(s, \lambda) \Delta s \\
& =\frac{1}{\lambda} \sin _{\lambda}(t, t) y^{\Delta}(t, \lambda)-\frac{1}{\lambda} \sin _{\lambda}(t, \rho(a)) y^{\Delta}(\rho(a), \lambda) \\
& -\left.\left(\cos _{\lambda}(t, s) y(s, \lambda)\right)\right|_{\rho(a)} ^{t}+\int_{\rho(a)}^{t}-\lambda \sin _{\lambda}(t, s) y(s, \lambda) \Delta s \\
& =-\frac{1}{\lambda} \sin _{\lambda}(t, \rho(a)) y^{\Delta}(\rho(a), \lambda)-y(t, \lambda) \\
& +\cos _{\lambda}(t, \rho(a)) y(\rho(a), \lambda)-\lambda \int_{\rho(a)}^{t} \sin _{\lambda}(t, s) y(s, \lambda) \Delta s
\end{aligned}
$$

and by the conditions (1.2) and (1.3), we get

$$
\frac{1}{\lambda} \int_{\rho(a)}^{t} \sin _{\lambda}(t, s) y^{\Delta \Delta}(s, \lambda) \Delta s=-y(t, \lambda)+\cos _{\lambda}(t, \rho(a))-\lambda \int_{\rho(a)}^{t} \sin _{\lambda}(t, s) y(s, \lambda) \Delta s .
$$

Therefore, considering (3.1) and (3.2) we have 


$$
y(t, \lambda)=\cos _{\lambda}(t, \rho(a))-\frac{1}{\lambda} \int_{\rho(a)}^{t}(q(s)+2 \lambda p(s)) y(s, \lambda) \sin _{\lambda}(t, s) \Delta s,
$$

where $\varpi(t)=1$. Differentiating (3.3) with respect to $t$, we obtain

$$
y^{\Delta}(t, \lambda)=-\lambda \sin _{\lambda}(t, \rho(a))-\frac{1}{\lambda} \sin _{\lambda}(\sigma(t), t)(q(t)+2 \lambda p(t)) y(t, \lambda)-\frac{1}{\lambda} \int_{\rho(a)}^{t} w(s) y(s, \lambda) \lambda \cos _{\lambda}(t, \rho(a)) \Delta s,
$$

or since $\sin _{\lambda}(\sigma(t), t)=\sin _{\lambda}(t, t)=0$, we have

$$
y^{\Delta}(t, \lambda)=-\lambda \sin _{\lambda}(t, \rho(a))-\int_{\rho(a)}^{t}(q(s)+2 \lambda p(s)) y(s, \lambda) \cos _{\lambda}(t, s) \Delta s .
$$

This completes proof.

\section{Conclusion}

Impulsive type diffusion eigenvalue problems appear in many fields of mathematical physics as quantum field theory, relativistics physics. Because of this importance, we notice that obtaining the solutions of these type problems on time scales will attract attention. In fact, our main goal is to obtain the asymptotic estimates for eigenvalues of this problem on $\mathbb{T}$. During the next period, we want to study Ambarzumyan's theorem and inverse problem for different type of operators on $\mathbb{T}$. We believe that this study will be an important step for this purpose.

\section{REFERENCES}

[1] S. Hilger, Ein Maßkettenkalkül vnit Anwendung auf Zentruvnsvnannigfaltigkeiten, [Ph.D. Thesis], Universitüt Würzburg, 1988.

[2] B. Aulbach, S. Hilger, A unified approach to continuous and discrete dynamics, qualitative theory of differential equations, Szeged (1988); Colloq. Math. Soc. János Bolyai, North-Holland, Amsterdam, 53 (1990), 37-56.

[3] M. Bohner and A. Peterson, Dynamic equations on time scales: an introduction with applications, Boston (MA), Birkhäuser, Boston Inc, 2001.

[4] F. M. Atici, G. Sh. Guseinov, On Green's functions and positive solutions for boundary value problems on time scales, J. Comput. Appl. Math. 141 (2002), 75-99.

[5] E. Bairamov, Y. Aygar and T. Koprubasi, The spectrum of eigenparameter-dependent discrete Sturm-Liouville equations, J. Comput. Appl. Math. 235 (16) (2011), 4519-4523.

[6] Y. Aygar and M. Bohner, On the spectrum of eigenparameter-dependent quantum difference equations, Appl. Math. Inf. Sci. 9 (4) (2015), 1725-1729.

[7] G. Sh. Guseinov, Self-adjoint boundary value problems on time scales and symmetric Green's functions, Turk. J. Math. 29 (2005), 365-380.

[8] C. J. Chyan, J. M. Davis, J. Henderson, W. K. C. Yin, Eigenvalue comparisons for differential equations on a measure chain, Electron. J. Diff. Equ. 35 (1998), 1-7. 
[9] R. P. Agarwal, M. Bohner and P. J. Y. Wong, Sturm-Liouville eigenvalue problems on time scales, Appl. Math. Comput. 99 (1999), 153-166.

[10] R. P. Agarwal, M. Bohner, D. O’Regan, Time scale boundary value problems on infinite intervals, J. Comput. Appl. Math. 141 (2002), 27-34.

[11] G. Sh. Guseinov, Eigenfunction expansions for a Sturm-Liouville problem on time scales, Int. J. Difference Equ. 2 (2007), 93-104.

[12] A. Huseynov and E. Bairamov, On expansions in eigenfunctions for second order dynamic equations on time scales, Nonlinear Dyn. Syst. Theory 9 (2009), 77-88.

[13] Y. Zhang and L. Ma, Solvability of Sturm-Liouville problems on time scales at resonance, J. Comput. Appl. Math. 233 (2010), 1785-1797.

[14] Q. G. Zhang, H. R. Sun, Variational approach for Sturm-Liouville boundary value problems on time scales, J. Appl. Math. Comput. 36 (1-2) (2011), 219-232.

[15] L. Erbe, R. Mert and A. Peterson, Spectral parameter power series for Sturm-Liouville equations on time scales, Appl. Math. Comput. 218 (2012), 7671-7678.

[16] E. Yilmaz, H. Koyunbakan and U. Ic, Some spectral properties of diffusion equation on time scales. Contemp. Anal. Appl. Math. 3 (2015), 238-246.

[17] İ. Yaslan, Existence of positive solutions for second-order impulsive boundary value problems on time scales, Mediterr. J. Math. 13 (4) (2016), 1613-1624.

[18] B. P. Allahverdiev, A. Eryilmaz and H. Tuna, Dissipative Sturm-Liouville operators with a spectral parameter in the boundary condition on bounded time scales, Electron. J. Diff. Equ. 95 (2017), 1-13.

[19] T. Gulsen and E. Yilmaz, Spectral theory of Dirac system on time scales, Appl. Anal. 96 (2017), 2684-2694.

[20] Y. Cakmak and S. Isık, Half Inverse problem for the impulsive diffusion operator with discontinuous coefficient, Filomat 30 (1) (2016), 157-168.

[21] M. Jaulent and C. Jean, The inverse s-wave scattering problem for a class of potentials depending on energy, Commun. Math. Phys. 28 (3) (1972), 177-220.

[22] A. Wazwaz, Partial differential equations: Methods and applications, Balkema Publishers, Leiden, 2002.

[23] F. G. Maksudov, M. M. Guseinov, A quadratic pencil of operators in the presence of a continuous spectrum, (Russian) Dokl. Akad. Nauk Azerbaidzhan SSR 35 (1) (1979), 9-13.

[24] M. G. Gasymov and G. Sh. Guseinov, Determination of a diffusion operator from the spectral data, Dokl. Akad. Nauk Azerbaijan SSSR 37 (2) (1981), 19-23.

[25] A. Fragela, Quadratic pencils of differential operators with integral boundary conditions, (Russian) Differential equations and their applications (Russian), 50-52, Moskov. Gos. Univ., Moscow, 1984.

[26] F. G. Maksudov, G. Sh. Guseinov, On the solution of the inverse scattering problem for the quadratic bundle of the one-dimensional Schrödinger operators on the whole axis, (Russian) Dokl. Akad. Nauk SSSR 289 (1) (1986), 42-46.

[27] E. Bairamov, Ö. Çakar and A. O. Çelebi, Quadratic pencil of Schrödinger operators with spectral singularities: discrete spectrum and principal functions, J. Math. Anal. Appl. 216 (1) (1997), 303-320.

[28] H. Koyunbakan, A new inverse problem for the diffusion operator, Appl. Math. Lett. 19 (10) (2006), 995-999.

[29] H. Koyunbakan, E. S. Panakhov, A uniqueness theorem for inverse nodal problem, Inverse Probl. Sci. Eng. 5 (6) (2007), $517-524$.

[30] H. Koyunbakan and E. Yilmaz, Reconstruction of the potential function and its derivatives for the diffusion operator, Z. Nat. forsch. A: Phys. Sci. 63 (3-4) (2008), 127-130. 
[31] C. F. Yang, Reconstruction of the diffusion operator from nodal data, Z. Nat. forsch. A: Phys. Sci. 65 (1-2) (2010), $100-106$.

[32] C. F. Yang and A. Zettl, Half inverse problems for quadratic pencils of Sturm-Liouville operators, Taiwanese J. Math. 16 (5) (2012), 1829-1846.

[33] R. Hryniv and N. Pronska, Inverse spectral problems for energy-dependent Sturm-Liouville equations, Inverse Probl. 28 (8) (2012), 085008.

[34] S. A. Buterin and C. T. Shieh, Inverse nodal problem for differential pencils, Appl. Math. Lett. 22 (2009), $1240-1247$.

[35] A. B. Yakhshimuratov, O. R. Allaberganov, The inverse problem for a quadratic pencil of Sturm-Liouville operators with a periodic potential on a half-axis, (Russian) Uzbek. Mat. Zh. 3 (2006), 96-107.

[36] I. M. Guseinov and I. M. Nabiev, The inverse spectral problem for pencils of differential operators, Sb. Math. 198 (11) (2007), 1579-1598.

[37] H. Koyunbakan, Inverse problem for a quadratic pencil of Sturm-Liouville operator, J. Math. Anal. Appl. 378 (2) (2011), 549-554.

[38] Y. P. Wang, The inverse problem for differential pencils with eigenparameter dependent boundary conditions from interior spectral data, Appl. Math. Lett. 25 (7) (2012), 1061-1067.

[39] R. Kh. Amirov, A. Nabiev, Inverse problems for the quadratic pencil of the Sturm-Liouville equations with impulse, Abstr. Appl. Anal. 2013 (2013), Art. ID 361989, 10 pp

[40] L. K. Sharma, P. V. Luhanga and S. Chimidza, Potentials for the Klein-Gordon and Dirac equations, Chiang Mai J. Sci. 38 (4) (2011), 514-526.

[41] K. Chadan, D. Colton, L. Paivarinta and W. Rundell, An introduction to inverse scattering and inverse spectral problems, SIAM, Philadelphia, PA, 1997.

[42] A. D. Orujov, On the spectrum of the quadratic pencil of differential operators with periodic coefficients on the semi-axis, Bound. Value Probl. 2015 (2015), Art. ID 117, 16 pp. 